\title{
Le lombricomposteur d'appartement, les déchets et la terre urbaine
}

Le cas de la Suisse romande

El lombricompostador en viviendas, desechos y la tierra urbana. El caso de la Suiza francesa

Home vermicompostors, waste and urban soil. The case study of Western Switzerland

Jacqueline Milliet

\section{OpenEdition} Journals

Édition électronique

URL : http://journals.openedition.org/ethnoecologie/2305

DOI : $10.4000 /$ ethnoecologie.2305

ISSN : 2267-2419

\section{Éditeur}

Laboratoire Eco-anthropologie et Ethnobiologie

\section{Référence électronique}

Jacqueline Milliet, « Le lombricomposteur d'appartement, les déchets et la terre urbaine », Revue d'ethnoécologie [En ligne], 8 | 2015, mis en ligne le 31 décembre 2015, consulté le 01 mai 2019. URL : http://journals.openedition.org/ethnoecologie/2305; DOI : 10.4000/ethnoecologie.2305

Ce document a été généré automatiquement le 1 mai 2019.

\section{(i) $\$$

Revue d'ethnoécologie est mis à disposition selon les termes de la licence Creative Commons Attribution - Pas d'Utilisation Commerciale - Pas de Modification 4.0 International. 


\title{
Le lombricomposteur d'appartement, les déchets et la terre urbaine
}

\author{
Le cas de la Suisse romande \\ El lombricompostador en viviendas, desechos y la tierra urbana. El caso de la \\ Suiza francesa \\ Home vermicompostors, waste and urban soil. The case study of Western \\ Switzerland
}

Jacqueline Milliet

«Quand je mets les mains sur le couvercle, ça c'est
ma mise à terre. Et je reste un moment comme

ça ».

Genève, le 25 novembre 2011

1 Maison des vers dans la maison des hommes, sur le balcon, à la cuisine, au salon ou au garage, le "lombricomposteur » trône sans arrogance, malgré des dimensions assez imposantes. Un modèle rond standard utile à une famille de quatre personnes désirant recycler ses déchets organiques elle-même mesure $60 \mathrm{~cm}$ de hauteur et $51 \mathrm{~cm}$ de diamètre. Depuis quelques années, les vers du compostage (Eisenia fetida et Eisenia andrei) sont les hôtes respectés des logements urbains et périurbains : ils y sont installés, nourris et élevés avec précaution. Ils profitent d'une place à eux dans la maisonnée et jouent un rôle dans l'économie du ménage.

2 En Suisse, la gestion des déchets figure dans la loi fédérale pour la protection de l'environnement du 7 octobre 1983 (RS 814.01, chapitre 4), et il incombe aux cantons de la mettre sur pied. Dès le début des années 1990, le pays figure parmi les premiers en Europe à encourager les citadins à composter leurs déchets organiques et à en organiser le ramassage ${ }^{1}$. Chacun a la possibilité de déposer son petit seau vert au bord du trottoir une fois par semaine, ou de remplir le grand conteneur brun de l'immeuble, vidé par le service de voirie pour le compostage ou la méthanisation ${ }^{2}$. Les cantons, les villes et les municipalités imposent de plus en plus le tri des déchets ménagers, le sac poubelle payant 
et la gestion des restes organiques produits par la famille s'organise en circuit fermé avec l'aide des vers.

\section{Enquête de proximité}

3 L'enquête sur l'acquisition et l'utilisation d'un lombricomposteur d'appartement a débuté en juin 2011. Le terrain ethnographique couvre l'arc lémanique ${ }^{3}$ (cantons de Genève et de Vaud) ainsi que le canton de Neuchâtel. Le choix répond à deux facteurs : c'est dans cette région qu'habite le chercheur, et que se trouve une éleveuse de vers qui est vraisemblablement la seule en Suisse, à faire de la lombriculture par andains ${ }^{4}$ (depuis 1986) (Figure 1). Dans sa petite exploitation de 19 hectares, elle a aménagé une dizaine de petits monticules linéaires, d'environ $90 \mathrm{~cm}$ de haut et d'une centaine de mètres de long chacun, composés d'écorce, de copeaux et de compost mélangés, dans lesquels les vers prolifèrent. Ils produisent de l'engrais ou «lombritonus » vendu aux garden centers et aux paysagistes des environs. En 2006, la fermière s'est lancée dans la promotion et la vente des « vermicaisses » d'intérieur et elle assure couvrir une grande partie du marché de la Suisse romande depuis 2013.

Figure 1 : Dans sa petite exploitation de 19 hectares, l'agricultrice produit du lombricompost dans une dizaine d'andains. Ce sont des tas d'environ $100 \mathrm{~m}$ de long, et d'environ $90 \mathrm{~cm}$ de haut. Ils sont constitués d'écorce, de copeaux et de compost mélangé, habitat dans lequel les vers prolifèrent. Les vers produisent de l'engrais ou comme elle l'appelle du « lombritonus ». Elle le vend aux garden centers et paysagistes des environs. Il semble qu'elle soit la seule en Suisse à faire de la lombriculture

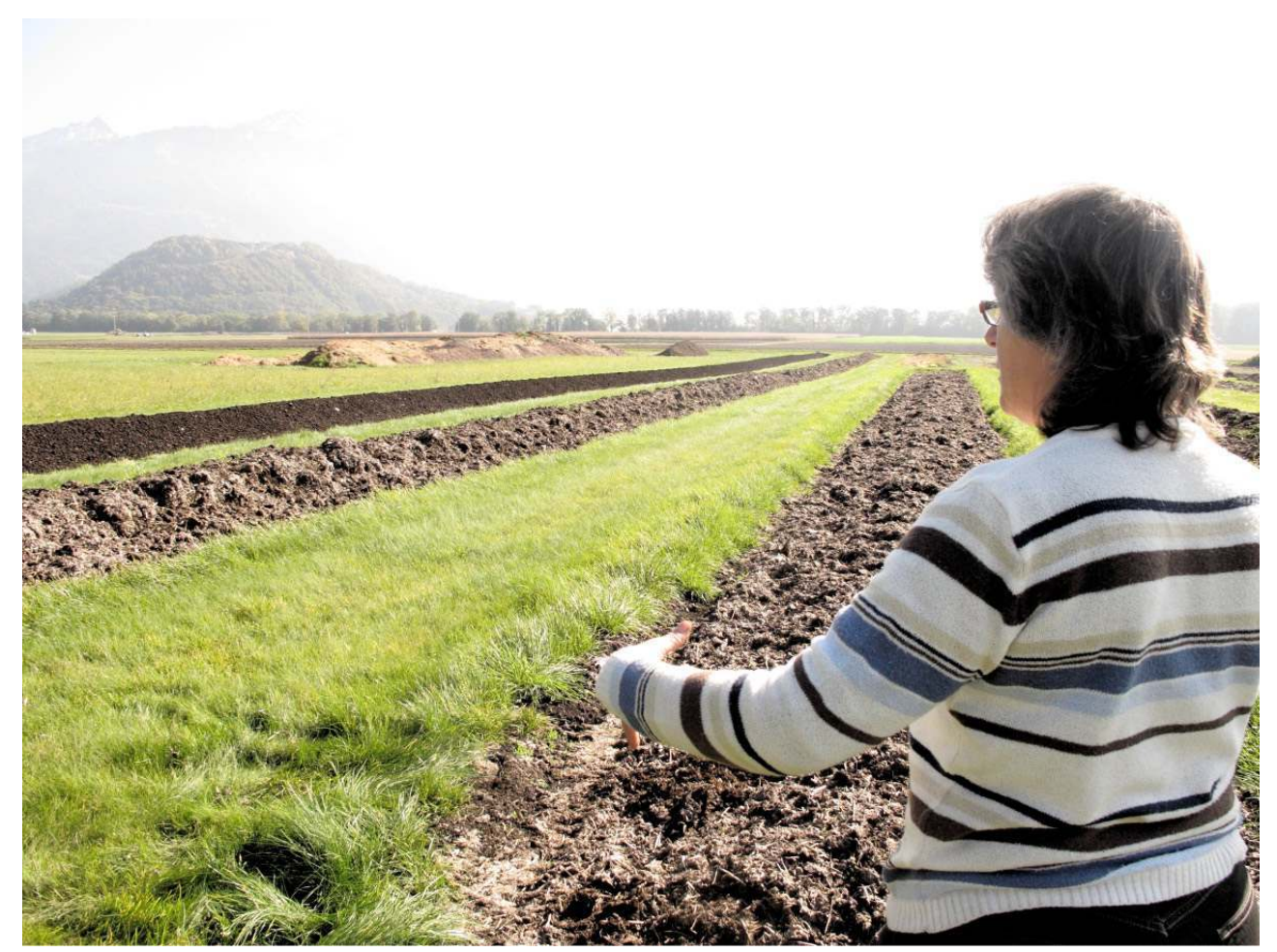

(C) Milliet 2011

Le lombricompostage domestique est une pratique peu visible et difficile à repérer. Pour atteindre ceux qui en font, l'enquête a été centrée sur la vermicaisse, excellent prétexte pour suivre les circuits de vente de la personne qui la commercialise et remonter ainsi 
jusqu'aux acquéreurs. La productrice de vers expose la marque australienne dont elle est la distributrice lors de salons de l'alimentation bio et des médecines naturelles et aux journées de jardinage et d'horticulture (Agrobiorama, Jardins en fête, Journée du printemps) qui ont lieu dans les grandes villes, les communes et les quartiers (Coppet, Vaumarcus, Nyon). Dans le cadre de la "politique verte", il a aussi été possible de remonter des filières et de rencontrer des élus en charge des départements environnement, enseignement, culture, éducation, des responsables des services publics " parcs et jardins » et de la voirie, des experts de l'application locale de l'Agenda $21^{5}$ et des représentants d $\mathrm{ACP}^{6}$ (Agriculture contractuelle de proximité, l'équivalent des AMAP $^{7}$ en France). La recherche a également porté sur le savoir des biologistes et des spécialistes de l'écologie rurale et des sols urbains, afin de retracer l'histoire de l'intérêt scientifique pour les vers de terre.

\section{Le système avec les vers}

5 Le lombricompostage est un procédé naturel de bio-oxydation qui transforme de la matière organique en "lombricompost", une matière très semblable à l'humus ou au terreau. Le processus s'enclenche sous l'action combinée des micro-organismes mésophiles (c'est-à-dire qui se développent le mieux à des températures modérées variant entre 25 et $40^{\circ} \mathrm{C}$ ) et des vers 8 . Le produit qui en résulte est souvent appelé « or noir» (désignation qu'on retrouve sur les sites promotionnels sur internet) car il est beaucoup plus riche en éléments nutritifs que l'engrais dû à d'autres méthodes, à la fermentation par exemple. Les agents biologiques responsables de ce recyclage et seuls capables d'être élevés sont deux vers épigés, l'Eisenia fetida (Savigny 1826) et l'Eisenia andrei (Bouché 1972) ${ }^{9}$, communément appelés "ver rouge», "ver tigré », "ver du fumier ", «ver de jardin ». Très ressemblants, ils se distinguent toutefois par une légère différence de couleur et de taille ${ }^{10}$. Dans la nature, ils vivent à la surface des sols, se nourrissent de pourriture de végétation, de compost et de fumier. Grâce à un système digestif caractérisé par une forte activité microbienne, ils mangent jusqu'à une fois leur poids par jour et réduisent le volume des déchets organiques de $40 \%$ à $60 \%$. Et $50 \%$ de ce qu'ils consomment ressort sous forme de déjections (le compost) (Adhikary 2012: 907-908). Assez élaboré, le tube digestif comprend une bouche, un pharynx qui peut servir de ventouse pour tirer les aliments dans les galeries et de broyeur pour les triturer (Figure 2). Les aliments passent ensuite dans le jabot où ils reçoivent un apport de carbonate de calcium des glandes de Morren, puis arrivent dans le gésier qui continue le broyage, et ils atteignent enfin l'intestin. Hermaphrodites, ces vers pondent des «capsules » qui éclosent au bout de trois semaines et donnent naissance à des petits à l'allure de fils blancs translucides qui prennent en quelques jours une coloration rose, puis rouge (Figure 3). Dans un environnement propice, ils deviennent adultes en deux ou trois mois et peuvent vivre trois ans. Sans yeux, sans oreilles, sans dents, ils n'émettent aucun son et ne dorment jamais. 
Figure 2 : Coupe latérale de la partie antérieure d'un lombric illustrant les organes internes, notamment le gésier et le jabot

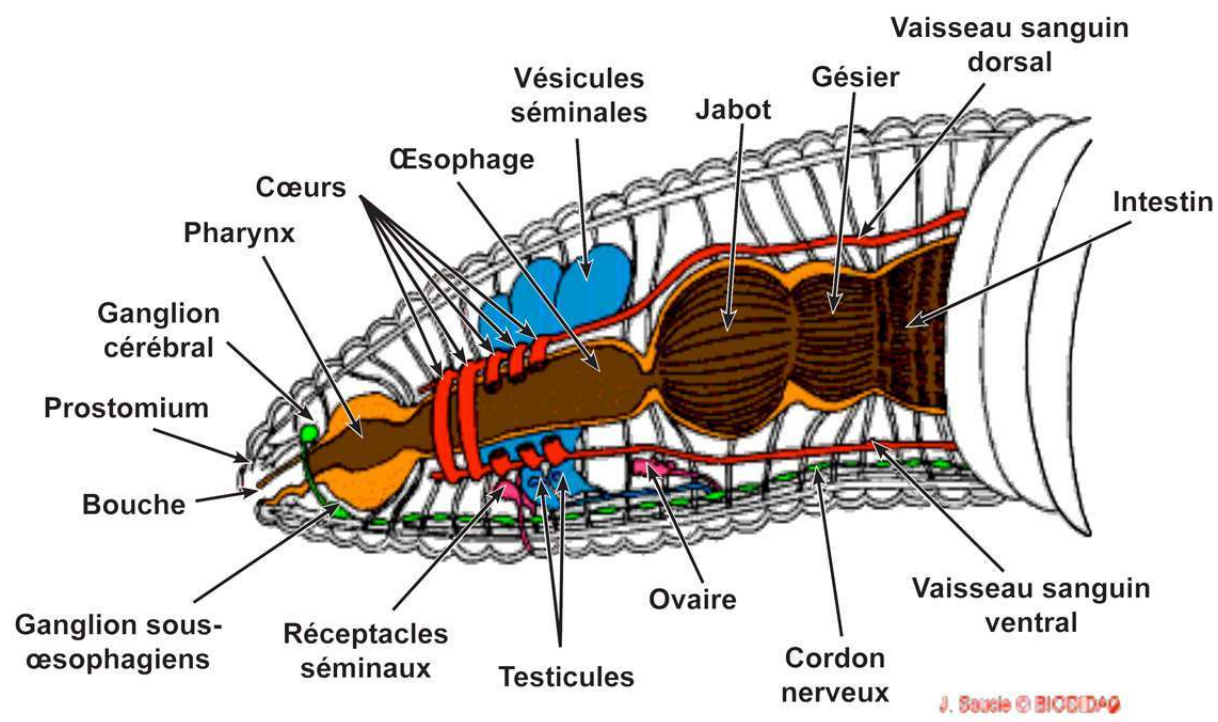

La forte activité microbienne du tube digestif des vers leur permet de manger jusqu'à une fois leur poids par jour. Ils réduisent le volume des déchets organiques de $40 \%$ à $60 \%$. Et $50 \%$ de ce qu'ils consomment ressort sous forme de déjections, donc de compost

(c) BIODIDAC. Internet simulium.bio.uottawa.ca, consulté le 28 juillet 2014

FIgURE 3 : Dans le composteur, les lombrics coexistent à différents stades de développement : d'œufs ou « capsules » donnant naissance à des petits à l'allure de fils blancs translucides qui prennent en quelques jours une coloration rose, puis rouge, aux adultes, plus violacés, mesurant de 60 à $90 \mathrm{~mm}$

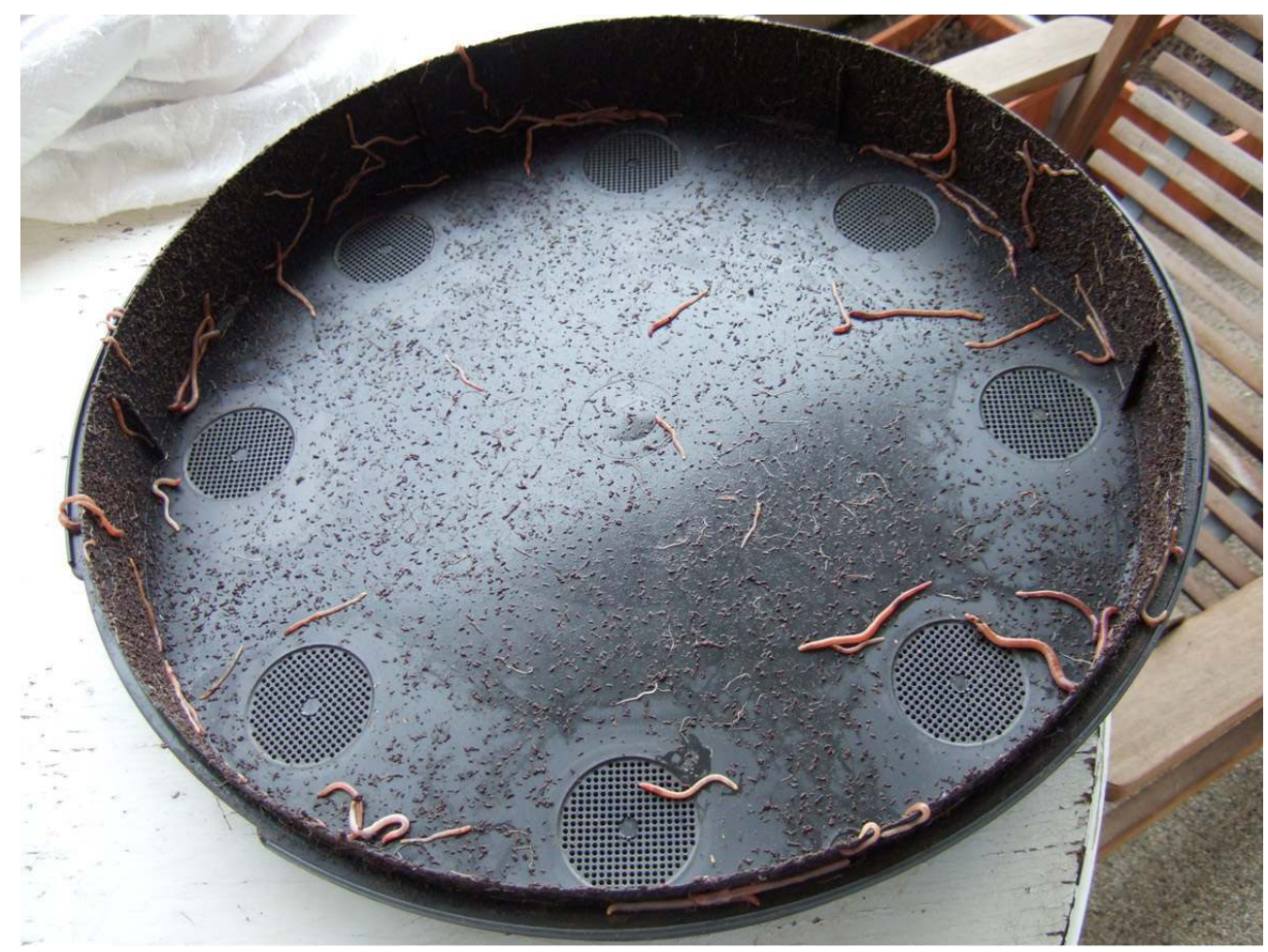

(c) Biro 2013 
FIgURE 4 : Chacun y va de son mode de protection contre le soleil, le froid, la pluie du lombricomposteur placé en plein air

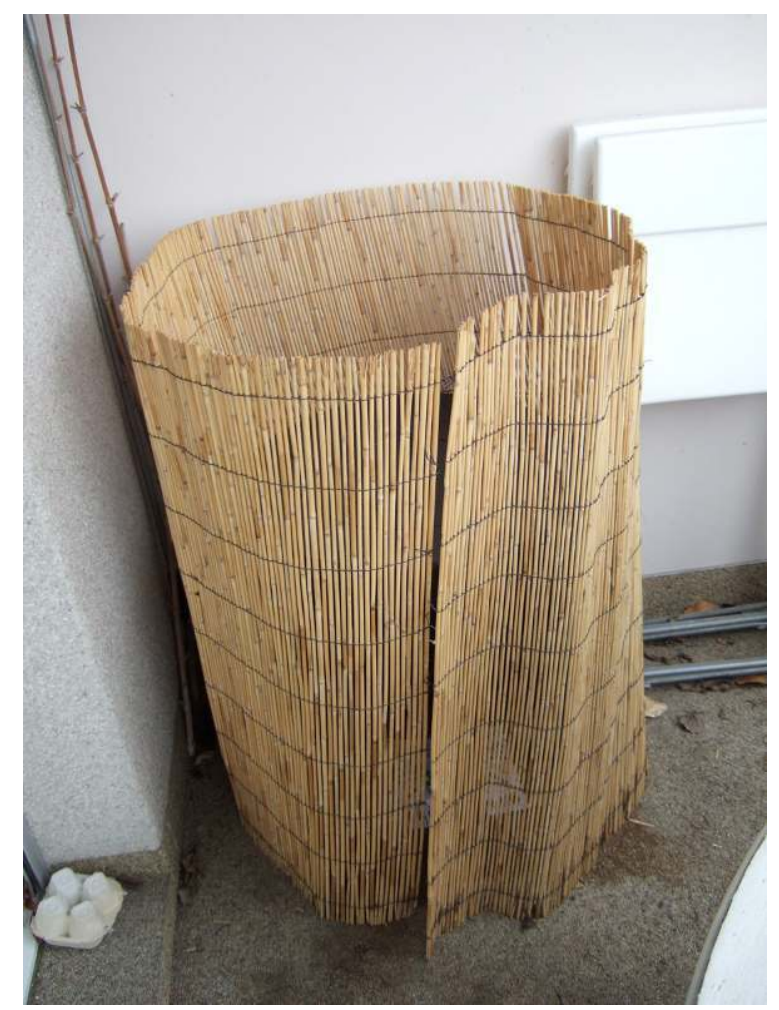

(c) Biro 2013

Sur le marché (surtout sur internet où les sites abondent), on trouve la maison des Eisenia sous cinq appellations différentes: lombricomposteur, lombricompostière, vermicomposteur, vermicompostière et vermicaisse. Les puristes n'utilisent pas le préfixe « lombri- », lui préférant celui de « vermi- » puisqu'il s'agit bien du travail d'un ver, qui n'est pas un lombric (Collaert 2009). Le terme «vermicompostage» vient de l'anglais vermicomposting ou worm composting. La langue française semble peu pressée de s'approprier ce terme. Peut-être parce qu'il sonne trop comme ver et vermine (Dumas 1996) ? Mais probablement aussi parce que la technique n'est pas connue chez nous depuis aussi longtemps que chez les Anglo-saxons. C'est aux États-Unis, au début des années 1970, que la biologiste et environnementaliste Mary Appelhof (1936-2005) a, la première, développé et commercialisé le Worm-a-way, organisé la vente de vers et écrit un best-seller Worms Eat My Garbage (1982), traduit en français en 2007 sous le titre « Les vers mangent mes déchets ». La "Worm Woman » accomplit à l'époque un important travail d'accompagnement dans les écoles et auprès des propriétaires de jardins. En Australie, le Can-O-Worm est breveté en 1994 et son utilisation est très populaire, comme en Nouvelle Zélande (Appelhof et al. 1996).

\section{1, « L'année du lombric »}

7 Quand l'enquête commence, c'est «L'année du lombric » et le ver arrive sur le devant de la scène. Pro Natura, ${ }^{11}$ une association suisse de protection des biotopes et espaces naturels, utilise chaque année un animal emblématique pour attirer l'attention de 
l'opinion publique sur une problématique. Cette année-là, le lombric est l'ambassadeur et le signal de la bonne santé des sols et incarne la menace que fait planer l'aménagement bétonneur du territoire. Lumbricus terrestris, "ver de terre commun » ou "ver de rosée ", agent majeur de la fertilité et de la vitalité des sols, voit son habitat diminuer de façon drastique : un mètre carré par seconde de terre disparaîtrait en Suisse sous les nouvelles constructions, routes, parcs de stationnement et industries (Pro Natura, communiqué de presse du 4 janvier 2011). Le choix d'un tel animal étonne. N'est-il pas plus un objet de dégoût? Réponse du responsable de l'information chez Pro Natura: "C'est vrai que le lombric est rébarbatif et provoque des réticences, mais il suscite aussi la curiosité. Nous avons donc joué sur l'élément de surprise, et cela a marché. $»^{12}$

8 En fait, le lombric n'est pas si impopulaire. Selon une employée du Jardin botanique de Neuchâtel, le public est intéressé par la terre « et par tout ce qui se passe en-dessous $~^{13}$ et la biologiste, spécialiste des sols urbains, confirme: "Les gens aiment la terre, maintenant aussi avec ses habitants $»^{14}$. Elle a pu observer elle-même l'inquiétude des passants pour les vers quand elle faisait des prélèvements d'échantillons dans les sols de Bâle en 2009. Elle rajoute : « Nous avons de la vraie terre à Bâle. Elle n'est fertile qu'avec une bonne faune de lombriciens, $c^{\prime}$ est la deuxième biomasse après les humains en ville ${ }^{15}$. C'est une vérité universelle, c'est la base de notre alimentation ». La ville n'est plus considérée sans sol aujourd'hui, alors que le substrat n'est constitué que de gravas et de terre de remblais (Graham 1999). La ville peut fabriquer de la terre, un potentiel de vie, dont les lombrics sont les ouvriers. La connaissance des sols (la pédologie et la pédogenèse) par l'étude des vers se développe en Suisse dès le milieu des années 1980, surtout après l'accident de Tchernobyl le 26 avril 1986 avec son nuage radioactif, et l'incendie du hangar du groupe pharmaceutique Sandoz (31 octobre 1996) provoquant une pollution de l'air et la contamination des eaux du Rhin à Bâle. Les biologistes avaient alors tiré la sonnette d'alarme. "Partout, les ruisseaux commençaient à mousser, les poissons crevaient, le mercure venait du Valais à cause d'Alusuisse, les sols et l'air étaient pollués (...) un bon sol doit avoir une certaine quantité de vers de terre » affirme l'auteur du guide d'Idenfication des Lombriciens de Suisse, G. Cuendet (2009) ${ }^{16}$. Une étude écotoxicologique du Plateau agricole suisse, de Genève à Saint Gall, est alors financée par les autorités fédérales et cantonales (Cuendet 1997). Il s'agissait d'évaluer l'état de la population des vers de terre, des anéciques (vers "ascenseurs» rosés), et dans une moindre mesure des endogés (vers «de profondeur» d'un blanc transparent), avec les bactéries associées, qui «font » les sols en mélangeant la matière organique de la litière végétale et les éléments minéraux du substrat ${ }^{17}$.

9 Avec l'active médiatisation du travail des biologistes et la « vedettarisation » du ver par Pro Natura, le lombricomposteur domestique et ses distributeurs commencent à bénéficier d'une certaine visibilité. À Vevey, ville de 18000 habitants au bord du Lac Léman, les responsables de l'Agenda 21 travaillent depuis une dizaine d'années au rapprochement de la ville et de la campagne en développant les produits « du terroir » et l'agriculture « contractuelle ». En 2011, ils mènent une action en faveur du fameux ver du compost (voir encadré). Les autorités encouragent alors les directeurs des écoles à s'équiper et la maison des lombrics devient un objet pédagogique de sensibilisation des enfants à la question du recyclage des déchets organiques. 


\begin{abstract}
Mesdames et Messieurs les représentants de la presse,
La Ville de Vevey offre une subvention de CHF 50 .- aux contribuables de la commune pour l'achat d'un lombricomposteur, lequel permet de réduire de $160 \mathrm{~kg}$ par personne la quantité annuelle de déchets devant être collectés.

Suite à la réorganisation du ramassage des déchets organiques dans les différents quartiers de la ville, l'Agenda 21 s'est interrogé sur une solution locale pour réduire les quantités de déchets transportés.

Sur les $\sim 500 \mathrm{~kg}$ de poubelles que chacun produit annuellement, environ un tiers pourrait être composté, c'est donc $\sim 160 \mathrm{~kg}$ de déchets par habitant qui n'aurait pas besoin d'être transporté au point de collecte, puis au lieu de compostage. II en résulte une économie de temps, d'argent et d'énergie.

Afin d'encourager les habitants de la commune à composter leurs déchets organiques directement chez eux, la ville de Vevey, dans le cadre de son Agenda 21, souhaite promouvoir le lombricompostage. II s'agit d'une solution simple consistant en un empilement de boîtes contenant quotidiens d'un ménage passent au travers des différents plateaux et sont transformés en un compost fertile.

Suivant la taille et le modèle choisis, un lombricomposteur coûte entre CHF 200 .- et 500 .- environ

L'Agenda 21 offre une subvention de CHF 50.- aux habitants, organismes de la commune souhaitant s'équiper pour composter leurs déchets organiques sur un balcon, dans une cuisine ou une cave. Les vers d'un lombricomposteur supportent facilement une absence de 2-3 semaines.

Pour obtenir une subvention, il suffit de s'adresser à l'Agenda 21 par téléphone ou courriel.
\end{abstract}

L'opération n'obtient pas un très grand succès. La personne qui en assure le suivi ne s'en montre pas très surpris : «On ne s'attendait pas à une explosion, c'est un processus qui vient à moyen terme. Aujourd'hui on doit être à 28 lombricomposteurs subventionnés. $»^{18}$ Il faut dire que le dispositif n'est pas bon marché. Un ménage de deux à trois personnes doit compter environ 300 euros pour un can-o-worm: «En plus il est importé d'Australie alors en termes d'écobilan..., sur notre site on donne quelques liens où en trouver des moins chers. $»^{19}$

\title{
Les boîtes à vers
}

Il est facile d'acheter un lombricomposteur sur la «toile ». C'est une boîte, généralement en plastique recyclé, parfois en bois, verticale ou horizontale, ronde ou rectangulaire, comprenant d'un à quatre étages. Mais le prix trop élevé, et surtout l'envie d'expérimenter incitent les adeptes à inventer toutes sortes de vermicaisses, à partir d'une variété étonnante de matériaux qu'ils recyclent : caisses de rangement Ikea, seaux de fleuriste pour les fleurs coupées, caisses de vin en bois, vieux tiroirs, bacs de poissonniers en frigolite, boîtes de conserve, etc. Une doctorante en biologie m'explique : «Mon lombricomposteur est entièrement en bois. Mon copain est ébéniste. Je voulais voir si ça marchait. Je connais personne qui en fait. Sur Youtube il y a beaucoup de vidéos qui disent comment faire. ${ }^{20}$

L'Association Glocal organise des animations de rues sur le thème du sol vivant et de la biodiversité, qu'elle combine avec des ateliers " de sensibilisation au lombricompostage » ${ }^{21}$. Le recyclage et « réapprendre » le travail manuel sont les motivations principales des participants. Le responsable du projet décrit les opérations :

" On prend un bac à poissons, il est sur trois étages. Il y a l'étage du bas qui permet de récupérer le jus de compost qui pourra être dilué avec de l'eau et réutilisé comme fertilisant. Ensuite il y a un étage intermédiaire où sont les vers. On leur 
donne de la nourriture qu'on ne mange pas et qu'on ne jette plus aux ordures ménagères. Les vers vont les manger, dès qu'ils auront fini ils passeront à l'étage supérieur par des interstices et des espaces qui leur permettent de passer d'un étage à l'autre. $»^{22}$

13 En somme, une caisse de $45 \times 30 \times 45 \mathrm{~cm}$ ou n'importe quel conteneur dont la base soit assez grande fait l'affaire. Il suffit de creuser des trous de drainage, sans oublier de prévoir un robinet pour récupérer le « jus de vers » ou « lombri-thé » qui, une fois dilué à $10 \%$, fait un excellent engrais.

14 S'il est acheté auprès de l'exportatrice de Can-o-Worm le colis est livré avec une petite poche en plastique : " J'y mets la matière active, constituée de 500 grammes de vers (200 à 250 animaux) et du substrat dans lequel ils ont été élevés à la ferme. ${ }^{23}$ La " matière active » n'est pas donnée non plus, une mère de famille s'offusque du prix : «Pour avoir un kilo de vers c'est 70 francs (environ 60 euros), c'est cher alors que ça se reproduit bien. On peut aller les chercher dans les tas de fumier $»^{24}$.

\section{Un système qu'il faut rôder}

Hors du contexte naturel, ce n'est pas si facile de s'en occuper. Il faut "s'impliquer", disent certains. Peut-être croyaient-ils qu'il suffisait de mettre des déchets et des vers ensemble ? Il faut en fait aménager un environnement adéquat qui constitue à la fois un milieu de vie et une source de nourriture. La marchande explique à tous ses clients comment faire :

« On parle de litière. C'est vraiment un lit au départ qui va leur servir de nourriture jusqu'à ce que les premiers déchets soient suffisamment décomposés pour qu'ils puissent les manger. Je leur dis vous mettez du journal mouillé au fond, de la fibre de coco bien mouillée et bien rincée et les lombrics dans leur substrat de base dans lequel ils sont élevés chez nous. Et ça ça fait une litière. Le substrat c'est l'environnement dans lequel ils sont élevés, dans nos litières de fumier à la ferme ». 25

16 La liste des matériaux utilisés pour créer une couche de 20 à $25 \mathrm{~cm}$ est hétérogène mais précise : terreau d'empotage, papier journal et carton déchiquetés (éviter le papier glacé et lourdement imprimé), coquilles d'œuf, sciure de bois. Certaines personnes recherchent le lien direct avec la nature pour y puiser des éléments. C'est ce qu'a fait cet enseignant avec sa famille: «On est allé chercher des feuilles dans la forêt. Et puis, ils sont comme des oiseaux, ils ont un gésier. Donc ils ont besoin de sable, on est allé en chercher au bord du Rhône ». ${ }^{26}$ Le sable facilite le broyage des matières avant qu'elles passent dans l'intestin.

17 Ces divers composants retiennent l'humidité, tout en étant assez légers pour assurer une aération. La «litière » doit être humide sans cependant dégoutter. Une fois lancé le processus de décomposition, la gestion du sec et de l'humide est importante. On ajoute des déchets et de la « litière », pour absorber les odeurs et faire obstacle à l'arrivée des mouches à fruits. Les rebuts organiques ne se jettent pas tels quels dans le composteur, il faut les préparer, les couper en petits morceaux. Plus ils sont découpés, plus ils présentent de surface de contact avec le milieu et plus leur décomposition est rapide (Le lombricompostage 2013). Certains doivent l'être plus finement que d'autres: les artichauts, les épluchures de pomme de terre, et les avocats forment un trop gros volume ou mettent plus de temps à se transformer. Il faut aussi éviter les oignons qui sentent fort avant la première phase de décomposition ou les feuilles de rhubarbe qui sont 
vermifuges. Les écorces d'agrumes, si elles ne sont pas enduites de paraffine, peuvent être ajoutées, mais en petite quantité car elles sont acides. Les vers aiment le marc de café, les sachets de thé, le pain et la pizza, le riz et les pâtes, ils détestent les résidus salés et vinaigrés. Il n'y a en principe pas d'odeur, mais les moucherons et les mouches viennent quand on met trop de fruits et de légumes à pépins (pommes, poires, tomate, courgette, concombre, courge, poivron).

\section{Les bergers des vers}

Pour obtenir que les vers travaillent, il faut les nourrir, connaitre ce qui est bon ou mauvais pour eux, bien trier et préparer les aliments. Il faut les mettre dans un milieu qui doit être clos pour éviter qu'ils ne s'échappent, il faut les protéger, faire en sorte qu'ils se reproduisent bien, que les vermisseaux ne fuient pas hors de la boîte, que l'équilibre de la population se fasse, etc. Il importe aussi de surveiller l'humidité, l'acidité, la température, l'aération, la lumière. Tous ces réglages fins, ce savoir-faire, font entrer les vers dans la catégorie des animaux d'élevage dont on attend un produit. Ces actions rappellent les trois exigences fondamentales auxquelles les humains doivent répondre pour qu'il y ait domestication : contrôle de l'alimentation, contrôle des déplacements et de la sécurité, et contrôle de la reproduction (Digard 1990). L'intervention sur la reproduction est minimale ici, mais elle est forte pour l'alimentation et les déplacements. Parfois l'élevage capote, faute d'avoir pris la mesure des actions nécessaires. Cette élue du Grand Conseil genevois m'explique :

«En fait ce qui s'est passé, je n'en suis pas très fière, les vers on eu beaucoup trop chaud. J'ai essayé de baisser la tente pour qu'ils aient pas de soleil direct. Je sais pas ce qui se passait mais le matin je retrouvais plein de vers sur mon balcon. Chaque matin je devais aller récupérer tout ça, j'ai essayé de voir ce que je pouvais faire, mais j'avais vraiment pas la place dans la cuisine, donc je me suis dit ils vont s'adapter, et puis en fait, ben progressivement ils sont tous morts $» .^{27}$

Les "lombri-composteurs" se voient comme des "bergers", des "éleveurs » et des "agriculteurs». Un enseignant qui travaille avec des jeunes en difficulté, raconte comment s'introduit « le processus du vivant en salle de classe » :

"C'est un moment merveilleux de déballer les vers. C'était juste incroyable. D'habitude on est devant des pages A4 [...]. Là on a vu ces animaux, et ça nous a complètement soudés. On a complètement changé de relations entre nous, on était autour de la table à couper l'emballage en petits morceaux. Commence alors l'épopée de l'élevage. Côtoyer le vivant, cela a donné toute une dynamique sociale dans la classe, parce qu'on devenait un peu les bergers du coin $»{ }^{28}$

Certains même s'entichent de ces petites bêtes, les faisant basculer du côté des animaux de compagnie. Cette femme habitant Lausanne prend quelque distance avec son attachement :

"Je fais rire mes amis avec mes vers. J'ai mis des photos sur Facebook. Alors je me dis que les vers c'est bien joli comme animal de compagnie, pour regarder la télé le soir, mes deux chats que j'ai depuis six mois, c'est quand même plus joli $»^{29}$.

\section{Terre à terre}

21 Les vers sont les agents d'un cycle qui restitue les déchets à la terre. Ils sont plus efficaces que le compost, qui ne réduit que de moitié le volume des déchets frais. Ils fabriquent de 
la terre, riche, fertilisante, qu'il est devenu impensable de perdre. La manière dont les villes gèrent les ordures est insatisfaisante. Une ancienne relieuse résume bien l'opinion qui tend à se généraliser :

"C'est quand même hallucinant qu'on fasse trier les déchets compostables et qu'on les incinère. C'est de nouveau consommer et produire des déchets quand ils les envoient au loin et que nous on n'a pas de retour $"{ }^{30}$.

Le retour, c'est l'« or noir ». Il est offert aux proches, aux riverains, à ceux qui ont un jardin ou des plantes vertes. La terre des vers crée de la fraternité en ville, des interactions avec les voisins, les amis. Avec elle, s'élaborent sur les balcons des potagers, des petits vergers, de véritables paysages campagnards en miniature suspendus, en pots. Ancien ingénieur dans un laboratoire de recherche médicale, en reconversion pour devenir conseiller environnemental indépendant, un jeune père de famille a commencé en 2008 à faire pousser des framboisiers, un cerisier et un abricotier sur sa terrasse :

«Mettre les déchets à la poubelle ça nous paraissait une aberration. Je voulais faire des plantations qui rapportent des fruits. Ça m'ennuie d'acheter de la terre. Aller la ramasser dans la nature c'est interdit. Je me suis dit on va composter nous-mêmes notre matière organique et puis on l'a réutiliserait ici. $»^{31}$.

Adepte inconditionnel, il convainc sa famille, ses amis, ses voisins :

" Ma mère, mon frère et sa copine, j'ai un copain qui en a offert un à sa mère, un autre qui en a construit un. J'en ai potentiellement une quinzaine en commande. Je fais la promotion de ce système $"$.

Il voudrait sensibiliser les élus de sa commune et s'inspirer de l'expérience de Vevey et la subvention. Un militant pour l'environnement genevois détaille ses préparations dans ces termes :

«J'ai acheté des bacsacs BACSAC ${ }^{32}$, je vais mélanger le terreau bio que j'ai acheté, mélanger le terreau traditionnel que j'ai aussi acheté et je vais mélanger avec mon vermicompost, je dois en avoir une douzaine de kilos, ça devrait permettre à mes fruits et légumes de bien pousser. $»^{33}$

D'autres développent une relation plus directe, plus physique : «Le lombricomposteur c'est donner accès à plein de choses. Le vivant. Pour moi le vivant ça passe par la terre ». L'ancienne relieuse artisanale explique pourquoi elle a quitté son job malgré de bonnes conditions salariales. Elle ressentait l'urgence de mettre les mains dans la terre. Quand elle se promenait dans la campagne les codes lui faisaient défaut pour comprendre le paysage :

« Est-ce que c'est une prairie d'herbes; est-ce que c'est planté ? Pourquoi on met les vaches là plutôt que là ? Pourquoi ces tas de bois, dont certains pourrissent et pas d'autres? Je me suis rendu compte que j'avais besoin de travailler avec mon corps à l'extérieur, de comprendre mon environnement, de pouvoir me situer.»

Membre d'une ACP au joli nom de "Lumière des champs ", elle démarche auprès des écoles de Vevey pour installer des lombricomposteurs afin de développer aussi la familiarisation avec la terre.

Les cours d'écoles sont en goudron, et s'il y a de la verdure, il est interdit de marcher dessus. Pour beaucoup d'élèves encore, «la terre c'est sale ». Pas question de se salir les mains, ni les chaussures :

« Il fallait passer dans l'herbe. Un élève a fait tout le tour du bâtiment. Il a dit moi je vais pas dans l'herbe avec mes shoes. C'est l'illusion d'être des créatures parfaites ", commente l'enseignant $»^{34}$. 
a port à la terre se modifie avec les générations. Dans la tranche d'âge entre six et dix ans, les enfants n'ont pas ou moins de préjugés que leurs aînés. Un membre de l'association Glocal le remarque souvent lors des animations de rue :

«Aux adultes ça semble normal que la terre soit morte, pas fertile, sans éléments organiques. De voir des bestioles dans la terre ça leur paraît pas normal. Tandis que les enfants quand on leur montre la terre avec les vers, au début ils sont un peu réticents, mais vite ils touchent sans problème. Les adultes, on l'a vu avec les enseignants, ils ont beaucoup de peine à mettre les mains dans le système, pour eux c'est pas propre. $\|^{35}$

L'animatrice veveysanne fait l'expérience des différences d'éducation entre les filles et les garçons : «Les garçons on les emmène volontiers dehors avec des bottes dans la boue et les filles pas forcément ». Et même s'ils ont une enfance originale, comme dans sa propre famille, un retour à l'ordre finit toujours par se produire :

"Avec mes enfants on a eu le contact avec la terre, avec le lombricompost, les fleurs, on a fait des plantes, on s'est roulé dans la boue, on s'est tartiné d'argile. Ils ont pas de problèmes à toucher un ver par exemple. Sauf ma fille, maintenant qui a sept ans et qui se confronte à la réalité des autres qui n'ont pas eu cette éducation, elle remet la distance. Elle touche plus un ver, c'est dégueulasse ${ }^{36}$

\section{Qui sont-ils ?}

Les personnes qui acquièrent ou fabriquent une vermicaisse sont encore minoritaires, faire le pas d'élever des vers chez soi n'est pas évident. Elles ne se reconnaissent pas dans une communauté et ne s'intègrent qu'individuellement dans des réseaux. Elles font partie du groupe des "alternatifs », dont les contours sont vagues. Elles se recrutent dans la classe moyenne et aisée, et dans la classe d'âge des 30 à 45 ans, autant les femmes que les hommes. Elles vivent en famille, mais aussi en couple ou seules. Dans un pays d'immigrations, (à Genève, plus de $50 \%$ de la population est étrangère) elles sont plutôt suisses, ou enfants d'immigrés (deuxième génération). Elles font preuve d'un engagement éco-responsable: (Figure 5) il ne suffit pas de se réclamer de la "pensée verte» (Boltanski \& Thévenot 1991) mais être capable de développer une stratégie individuelle de réduction à la source des déchets et de recyclage. Avec la terre produite en faisant disparaître les rebuts de cuisine et en élevant des vers, tous se voient en " paysans » et se rapprochant de la réalisation d'un rêve d'autosuffisance alimentaire, la plus bio possible. Ils entretiennent une affinité symbolique avec l'agriculture et inventent un nouveau territoire, la terre urbaine, dont les ramifications comprennent des liens plus ou moins serrés avec les pratiques agricoles de proximité ou contractuelles. 
FigURE 5 : Kit pour démarrer un composteur, prêt à être posté : $500 \mathrm{gr}$ de lombrics dans un peu de substrat de base dans lequel ils ont été élevés, de la fibre de coco ou « litière » qui va servir de nourriture jusqu'à ce que les premiers déchets soient suffisamment décomposés

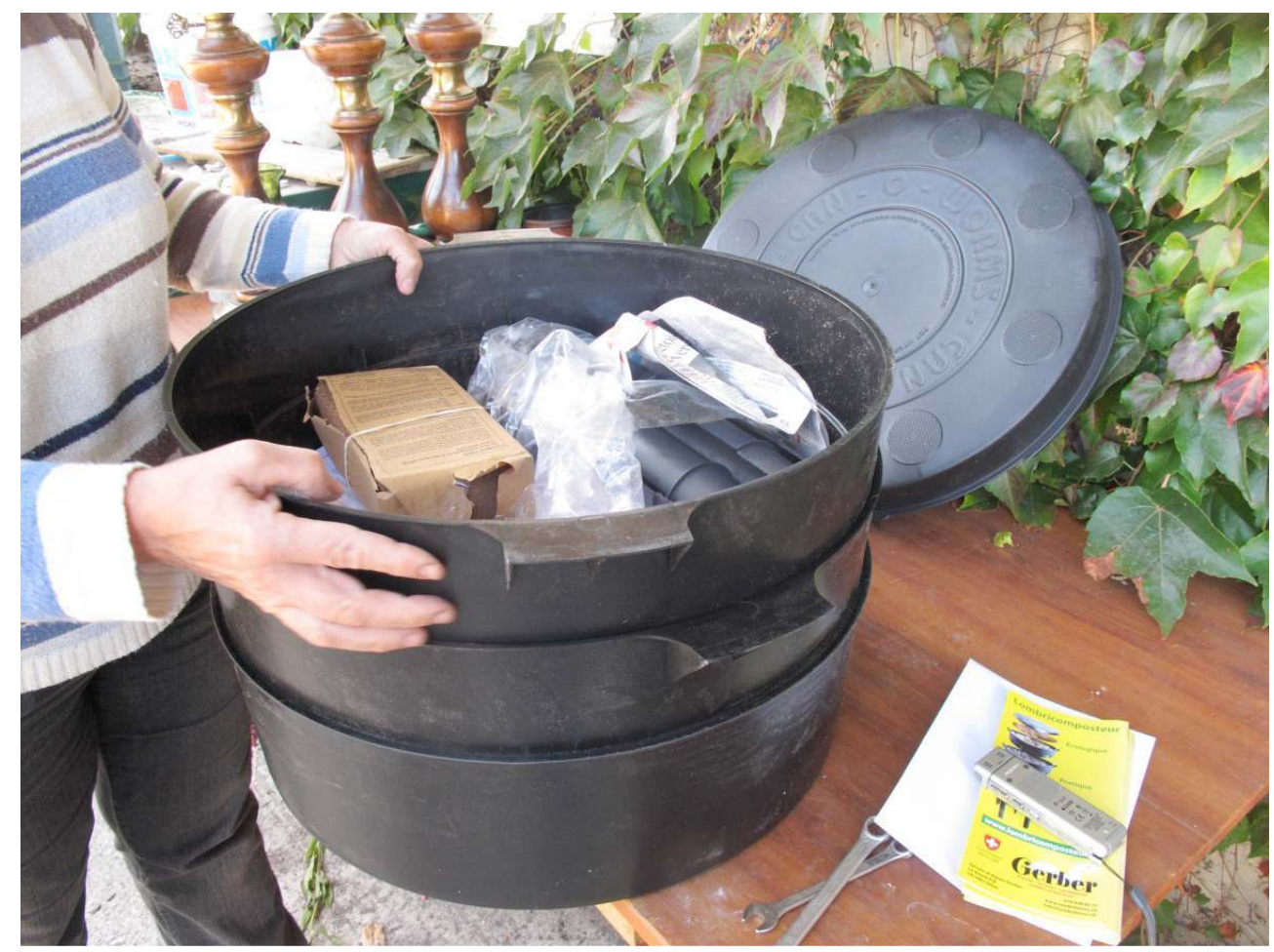

(C) Milliet 2011

32 L'inlassable activité des vers les fascine. Ces petits soldats, on se met à les aimer. Mais c'est le processus de la décomposition qui subjugue vraiment, parce qu'il est autonome et tout près de soi : « Il y a des milliers de choses qui se passent et qui se créent. Tout-à-coup y a des champignons qui poussent alors qu'on les a pas mis là ", s'émerveille l'ancienne relieuse. Sachant qu'il faut trois à quatre mois pour passer des déchets à l'or noir, la perception du temps change: "Dans un monde où on veut aller vite», le recyclage impose un rythme différent, un temps plus lent et « le lombricomposteur, ça va assez long en fait, c'est ce que j'aime. On a pas tout, tout de suite ». Dans une société où les modes de vie s'organisent autour de la mobilité, le temps de la décomposition opérant dans ce microcosme fonctionne symboliquement comme l'épicentre de la maison qui permet de s'ancrer au monde : « Quand je mets les mains sur le couvercle, ça c'est ma mise à terre. Et je reste un moment comme ça ", dit une cliente à son fournisseur ${ }^{37}$. S'imaginer sentir la vibration de l'activité des vers sous ses mains, cela pacifie et sécurise, délimite un territoire pour les urbains qui ont l'impression de vivre complètement hors-sol. Cet enseignant a choisi de s'éloigner de la grande ville où il travaille, il habite une maison dans un village au pied des Alpes vaudoises : « Cela permet de renouer avec quelque chose de rond. On a mangé nos carottes, le reste vous leur donnez à manger et puis après je vais mettre ça dans mon jardin. $Y$ a pas de déchets. Il y a quelque chose d'harmonieux. Ça me renvoie aussi à ma finalité. Les vers vont aussi me bouffer et je vais finir comme ça $»^{38}$. Avant de livrer son cadavre aux vers, cet orphelin de la terre (comme il aurait pu se définir lui-même), pousse la logique du recyclage jusqu'à se fabriquer des toilettes sèches au fond du jardin : 
«Pour moi c'est de l'ordre de l'évidence, je ne vais pas faire ma crotte dans l'eau pour nettoyer l'eau cinquante mètres plus loin. C'est très rassurant, c'est une manière d'être en lien avec soi et d'interagir avec le vivant ${ }^{39}$.

Quelles que soient leurs fonctions - élimination de la structure végétale et animale -, les vers (ce ne sont pas les mêmes) épurent les restes de la vie pour «booster ", fertiliser un nouveau départ.

Élever des vers chez soi, c'est s'intégrer au cycle complet du recyclage, en harmonie avec le vivant menacé par l'exploitation globalisée de la planète. Quels autres animaux seraient capables de procurer de la terre à domicile, nécessaire au besoin d'enracinement dont chacun parle à sa manière?

C'est sans doute ce qu'a sous-estimé une équipe d'architectes de Nantes, qui entendait instrumentaliser le travail des lombrics sous une forme hygiéniste afin de les intégrer dans les habitations. Conscients qu' « à moins d'être un tout petit peu militant, les vers dans la cuisine culturellement c'est pas tout à fait acceptable », ils proposaient de rendre le lombricomposteur invisible en le logeant dans la paroi de la cuisine au même titre que les récipients des divers déchets triés : (Figures 6 et 7 composteurs dans la maison (bien visible, et caché))

«On va moderniser le principe de sorte qu'il va se vider tout seul. Sous forme d'un tourniquet, un petit peu comme notre appareil digestif, on a une entrée, un tiroir, où on met les déchets et une sortie sous forme de compost. Sorte de tambour qui met quatre mois pour faire un tour complet. On a des alvéoles, qui sont des tôles perforées qui permettent aux lombrics de passer au travers et d'aller toujours manger le frais $»^{40}$.

Un tel arrangement parait peu réalisable. On a vu toutes les contraintes du milieu dont il fallait tenir compte (aération, humidité, lumière, température). Mais surtout, sans contact avec les vers et la décomposition, bien des personnes n'y trouveraient pas le réconfort qu'elles recherchent. 
FigURE 6 : Sur les balcons s'élaborent des potagers, des petits vergers, de véritables paysages campagnards en miniature suspendus en pots. Les vers sont les agents d'un cycle qui restitue les déchets à la terre. Ils fabriquent de la terre, riche et fertilisante

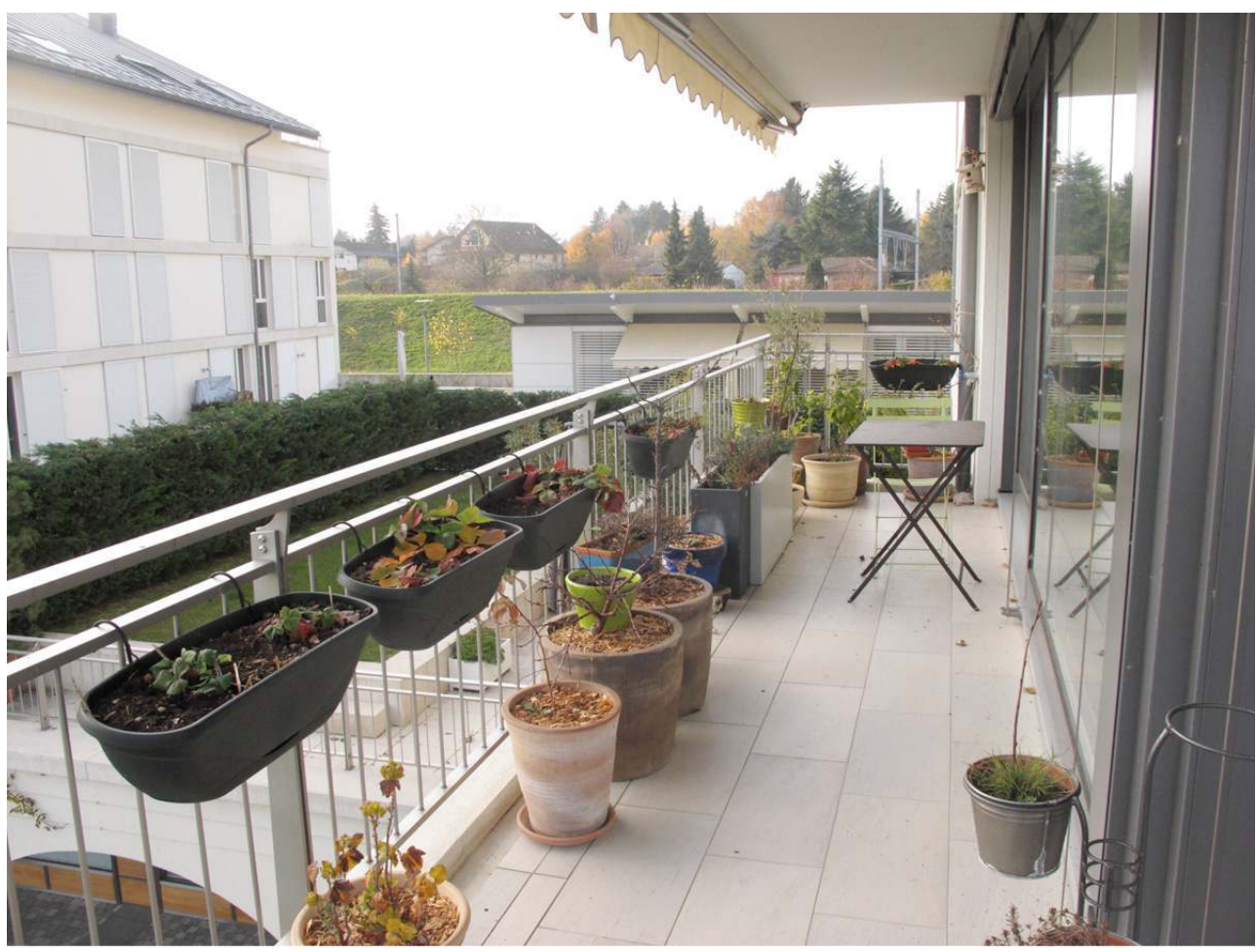

(c) Milliet 2011

\section{Un signe des temps}

Très récente en Suisse, la pratique du lombricompostage domestique se répand de plus en plus, grâce à une publicité soutenue sur internet et dans des salons locaux de jardinage ou de médecines naturelles. Selon un membre de l'association Glocal qui organise des animations de rues et pédagogiques dans des communes intégrées au tissu urbain genevois, elle commence «à faire boule de neige ». On y apprend avec lui à confectionner et gérer «le système ", les élèves rentrent à la maison avec un pot rempli de terreau et une graine plantée dedans : sensibilisation à la terre et à son potentiel vivant pour les enfants, conscience écologique du recyclage pour les adultes. Les services publics soutiennent financièrement ce genre d'actions, « de l'ordre de 400 francs par animation, ça permet de répondre aux coûts, d'avoir une petite marge au cas où le matériel était plus cher que prévu $»^{41}$. À Vevey, la ville de la fameuse subvention, le responsable de l'Agenda 21 s'oriente aussi vers les écoles. Au fil de l'enquête, des noms de nouveaux adeptes qui font référence sont donnés, une conseillère municipale qui fait partie de la commission du développement durable, un membre du Grand Conseil (parlement cantonal) actif à la commission parlementaire de l'enseignement, de l'éducation et de la culture. Et il y en a d'autres. La dépositaire du can-o-worm australien confirme l'élan d'intérêt, ses ventes auraient doublé entre 2011 et 2014. Elle repère l'émergence d'une mode : " Y en a qui m'ont dit, c'est les nouveaux tamagotchi ${ }^{42}$ ! Je leur ai dit alors non, le but c'est pas de les nourrir eux, mais de vous nourrir. C'est des animaux de compagnie si on veut mais qui rendent service ${ }^{43}$. Le lombricomposteur collectif pourrait se développer, au niveau de 
l'immeuble, du quartier, de la municipalité. Une commune de Genève en a étudié le projet, puis l'a abandonné. Trop de problèmes d'organisation se posent encore : qui s'en occupe, qui le gère, une association, un concierge, la voirie, la commune ? Les produits du développement durable, du «bio», et du recyclable sont en pleine expansion. C'est un paradoxe alors que nous vivons une période économique problématique et que les attitudes de consommation se recentrent sur la simplicité, voire la frugalité, et la convivialité (Caillé et al. 2011). Sur " la toile », il y a pléthore d'équipements domestiques, «naturels » et simples, abritant et organisant l'exploitation des vers. On a déjà vu qu'un grand choix de compostières y est proposé. Arrivent les toilettes sèches à lombricompostage qui récupèrent les excréments pour en faire du compost. Voilà une terre urbaine bien originale qui oblige à faire reculer les frontières du dégoût déjà évoqué pour les vers.

\section{BIBLIOGRAPHIE}

Adhikary S. 2012 - Vermicompost, the story of organic gold: A review. Agricultural Sciences 3 (7) : 905-917.

Appelhof M. 1982 - Worms eat my garbage. Kalamazoo, Michigan, USA, Flower Press, 162 p.

Appelhof M., Webster K. \& Buckerfield J. 1996 - Vermicomposting in Australia and New Zealand. Biocycle 37 : 63-66.

Boltanski L. \& Thevenot L. 1991 - De la justification. Les économies de la grandeur. Paris, Gallimard, 485 p.

Collaert J.-P. 2009 - Lombricompost pour tous. Passez au ver pour vos déchets. Aspet, éditions de Terran, 128 p. (Jardinier nature)

Cuendet G. 1997 - Peuplements lombriciens des prairies permanentes du Plateau suisse. Rapport de synthèse, Office fédéral de l'environnement, des forêts et du paysage (OFEFP), 90 p. (Cahier de l'environnement 291)

Cuendet G. 2009 - Identification des Lombriciens de Suisse. Vauderens (Suisse), Zoocontrol 1, 21 p.

Digard J.-P. 1990 - L'homme et les animaux domestiques. Anthropologie d'une passion. Paris, Fayard, 325 p.

Dumas M. 2010 (1996) - Les vers. Des croyances populaires au lombricompostage. Austin (Québec), éditions Berger, $156 \mathrm{p}$.

Duvigneau P. \& Denaeyer de Smet S. 1977 - L'écosystème urbain Bruxellois. In Duvigneau P. \& Kostemont P. (Ed.) Productivité biologique en Belgique, Scope, Travaux de la Section belge du Programme Biologique International : 608-614.

Graham E. 1999 - Exploitation du cadre bâti à des fins agricoles. In Mustafa Koc (Ed.) Armer les villes contre la faim. Systèmes alimentaires urbains durables. Sous la direction de, Rod MacRae, Luc J.A. Mougeot et Jennifer Welsh, CRDI, Canada : 161-166 
Le lombricompostage 2013 - Une façon écologique de traiter les résidus organiques. Guide pratique, Ville de Vevey, $20 \mathrm{p}$.

Philippot V. 2010-1011 - Approche ethnologique de la pratique du compostage collectif citadin. Les vertus éco citoyennes à l'épreuve de l'enquête. Mémoire de Master, Paris, Muséum national d'histoire naturelle, $97 \mathrm{p}$.

\section{NOTES}

1. «Les cantons encouragent la valorisation des déchets compostables par les particuliers euxmêmes, notamment par le biais d'informations et de conseils. (...) Si les particuliers n'ont pas la possibilité de valoriser eux-mêmes leurs déchets compostables, les cantons veillent à ce que les déchets soient dans la mesure du possible collectés séparément et valorisés. » (Ordonnance du 10.12.1990 sur le traitement des déchets, OTD, RS 814.600 article 7).

2. «La méthanisation (encore appelée digestion anaérobie) est une technologie basée sur la dégradation par micro-organismes de la matière organique en conditions contrôlées et en l'absence d'oxygène (réaction en milieu anaérobie contrairement au compostage qui est une réaction aérobie). Cette dégradation aboutit à la production d'un produit humide, riche en matière organique et partiellement stabilisée, appelé digestat et de biogaz ». http:// www2.ademe.fr/servlet/KBaseShow?sort=-1\&cid=96\&m=3\&catid=15555, consulté le 11.11.2015

3. Aire urbaine située autour du lac Léman avec deux pôles principaux, Genève/Lausanne, Vevey/Montreux.

4. Voir le site internet http://www.lombritonus.ch/.

5. L'Agenda 21 est un plan d'action pour le xxI ${ }^{\mathrm{e}}$ siècle adopté par 173 chefs d'État lors du Sommet de la Terre, en 1992.

6. L'Agriculture contractuelle de proximité (ACP) «lie par contrat des consommateurs-trices et un ou des producteurs-trices d'une région définie pour un approvisionnement de produits alimentaires. (....) En échange de ces produits, cultivés dans le respect de l'environnement, le consommateur s'engage à payer ces produits à l'avance et généralement à participer quelques fois par année à divers travaux sur l'exploitation (production, transformation, distribution) ». FRACP, Fédération romande d'agriculture contractuelle de proximité. http://www.acpch.ch, consulté le 04.04.2013).

7. Une AMAP (association pour le maintien d'une agriculture paysanne) naît en général de la rencontre d'un groupe de consommateurs et d'un producteur prêts à établir entre eux un contrat pour une ou deux saisons de production (printemps/été et automne/hiver). «En achetant leur part de production à l'avance, les consommateurs garantissent un revenu au paysan. L'AMAP participe ainsi au maintien d'une agriculture de proximité et à la gestion de la pression foncière » (site national des AMAP, www.reseau-amap.org/, consulté le 14.10.2012).

8. Le processus est actif entre $10^{\circ} \mathrm{C}$ et $32^{\circ} \mathrm{C}$, non pas à la température ambiante, mais à celle de la couche humide du matériel organique.

9. C'est le naturaliste français Marie-Jules César Lelorgne de Savigny (Provins 1777-Versailles 1851), collègue de Lamarck et Cuvier au Muséum national d'histoire naturelle (Collaert 2009: 13), quia identifié et nommé le genre Eisenia, en 1924.

10. L'Eisenia fetida est rouge violacé et possède des anneaux clairs, presque jaunes. Sa longueur est de 60 à $90 \mathrm{~mm}$ (poids moyen $300 \mathrm{mg}$ ). L'Eisenia andrei se caractérise par sa coloration rouge vineux uniforme et par une longueur généralement plus petite, 50 à $80 \mathrm{~mm}$ (poids moyen $250 \mathrm{mg})$.

11. Fondée en 1910, Pro Natura est active avec ses sections dans tous les cantons suisses et gère plus de 600 réserves naturelles et une douzaine de centres nature. 
12. Entretien du 18 janvier 2012.

13. Entretien du 2 septembre 2011.

14. Entretien du 13 septembre 2011.

15. La biomasse équivaut à la «masse totale de l'ensemble des êtres vivants occupant, à un moment donné, un biotope bien défini ». (http://www.larousse.fr/dictionnaires/francais/ biomasse/9439, consulté le 28.04.2013). La biologiste se réfère à l'ouvrage de Duvigneaud et Denaeyer de Smet (1977).

16. Entretien du 22 septembre 2011.

17. Ce ne sont pas les mêmes vers que ceux du compost, les épigés, vers "de surface " qui se nourrissent de la litière déjà fragmentée par les anéciques (creusant de profondes galeries verticales) et les endogés (creusant des couloirs horizontaux) (Cuendet 1997).

18. Entretien du 28 octobre 2011, entretien du 30 avril 2013.

19. Entretien du 30 avril 2013.

20. Entretien du 16 décembre 2011.

21. Association créée en 2010 et localisée à Lancy dans le canton de Genève, dont les finalités sont de promouvoir le développement durable. (http://associationglocal.wordpress.com/educationau-developpement-durable/ consulté le 03.05.2013).

22. Entretien du 2 mai 2013.

23. Entretien du 17 octobre 2011.

24. Entretien du 16 décembre 2011.

25. Entretien du 24 mars 2013.

26. Entretien du 21 décembre 2011.

27. Entretien du 25 avril 2013.

28. Entretien du 21 décembre 2011.

29. Entretien 1er avril 2013.

30. Entretien 16 décembre 2011.

31. Entretien du 25 novembre 2011.

32. Le $\mathrm{BACSAC}^{\circledast}$ est un « contenant souple, de différents formes et grandeurs, pour la culture hors-sol. Sa double paroi en géotextile, $100 \%$ recyclable, assure l'équilibre entre l'air, la terre et l'eau » (http://www.bacsac.fr/fr/concept/, consulté le 04.05.2013).

33. Entretien du 2 mai 2013.

34. Entretien du 22 décembre 2011.

35. Entretien du 2 mai 2013. Sur les attitudes face à la terre, au compost et aux vers, voir Philippot 2010-2011.

36. Entretien du 16 décembre 2011.

37. Entretien du 25 novembre 2011.

38. Entretien du 21 décembre 2011.

39. Entretien du 21 décembre 2011.

40. Tiré de l'émission de Ruth Stegassy Terre à Terre sur France Culture, le samedi de $7 \mathrm{~h}$ à $8 \mathrm{~h}$, le 12 novembre 2011 : «Les questions d'environnement mises à plat pour mieux penser les ressorts et les implications ». Exemple de Nantes. Concepteurs et architectes : Laurent Lebeau et Victor Massy.

41. Entretien du 30 avril 2013.

42. «Un Tamagotchi est un animal de compagnie virtuel japonais. Ce nom est un mot-valise créé à partir des mots «œuf " (tamago) et «montre " (wotchi). Le jeu consiste à simuler l'éducation d'un animal à l'aide d'une petite console miniature, de la taille d'une montre, dotée d'un programme informatique » (http://fr.wikipedia.org/wiki/Tamagotchi, consulté le 13.05. 2013).

43. Entretien du 7 octobre 2011. 


\section{RÉSUMÉS}

Les autorités suisses imposent de plus en plus le tri des ordures ménagères, le sac poubelle payant et la gestion des déchets organiques. Leur récupération commence à s'organiser en circuit fermé, avec l'utilisation de vers (Eisenia fetida et Eisenia andrei). Hôtes respectés des logements urbains et périurbains, installés dans des «maisons» appelées "lombricomposteur", « lombricompostière ", " vermicomposteur ", " vermicompostière » et "vermicaisse ", ils sont nourris et élevés avec précaution. Ils fabriquent de la terre, riche, fertilisante, qu'il est devenu impensable de laisser perdre. Les résultats du terrain ethnographique montrent qu'une telle production en ville implique l'organisation de nouvelles connaissances techniques, de nouvelles solidarités, de nouvelles manières de vivre la nature en milieux urbains.

Las autoridades suizas, hacen necesarias la clasificación de los residuos domésticos según el tipo, las bolsas de basura sujetas a pago, y la gestión de los desechos orgánicos. Su recuperación empieza a organizarse en circuito cerrado, utilizando gusanos (Eisenia fetida et Eisenia andrei). Invitados respetados en las viviendas urbanas y suburbanas, instalados en casitas llamadas « lombicompostador ", « vermicompostador », "compostera de lombrices ", son alimentados y criados con precaución. Producen une tierra fertilizante, muy rica, que no se puede perder. Los resultados de la investigación etnográfica demuestran que tal producción urbana implica la organización de nuevos conocimientos técnicos, de nuevas solidaridades, de nuevas maneras de vivir la naturaleza en la ciudad

Swiss authorities are becoming stricter and stricter about imposing the sorting out of domestic waste, the prepaid bin bags as well as the handling of organic leftovers whose organised recycling begins in a closed circuit with the use of worms (Eisenia fetida et Eisenia andrei). These creatures are highly respected guests in city centre and suburban housing, where their small homes named "vermicompost containers ", "vermicompostors " are found. Fed and raised with care, these worms produce fertile soil and has become unthinkable to dispose of. The results of anthropological fieldwork shed light on this new urban production involving the organisation of new technical know-how, new solidarity, and new ways of experiencing nature in a city environment.

\section{INDEX}

Mots-clés : terrain ethnographique, vers de terre, élevage, ville, déchets

Palabras claves : investigación etnográfica, gusanos, cría, ciudad, desechos

Index géographique : Suisse

Keywords : anthropological fieldwork, earthworm, husbandry, city, waste 


\section{AUTEUR}

\section{JACQUELINE MILLIET}

UMR 7206, CNRS-MNHN-UMPC Eco-Anthropologie et Ethnobiologie, Paris, http://

www.ecoanthropologie.cnrs.fr

Calle Atarazana vieja 12, 18010 Granada, España

jacq.milliet@gmail.com 